\title{
Green Bank dish may be last of the giants
}

\section{Colin Macilwain, Washington}

The largest steerable, single-dish radiotelescope ever built will be dedicated this week at Green Bank, West Virginia, in a ceremony to be attended by astronomers, politicians and research agency chiefs.

But the gargantuan Green Bank Telescope (GBT) - which is already six years late and tens of millions of dollars over budget may mark the end of an era, as astronomy migrates from single-dish radiotelescopes to arrays of smaller instruments.

The 110-metre-diameter GBT has a unique reflective dish that sends radio waves from its field of observation to a receiving tower. This tower is offset from the dish itself, and so does not obstruct the telescope's field of view. The dish consists of an array of 2,000 panels, designed to move in real time to compensate for thermal or gravitational flexing of the structure and so keep the instrument sharply focused.

"It is an extraordinary instrument even if we've been waiting for it a little bit longer than we had hoped," says Joseph Taylor, an astronomer at Princeton University in New Jersey, who won the 1993 Nobel prize for physics.

For Taylor and other pulsar astronomers, the GBT will offer an opportunity to conduct low-frequency observations of great sensitivity as soon as observation time on it becomes available, probably next spring.

But even as Senator Robert Byrd (Democrat, West Virginia) declares the telescope open, questions of finance remain unanswered. Comsat, the contractor responsible for the telescope's construction, has yet to hand it over to the National Radio Astronomy Observatory (NRAO), the arm of the National Science Foundation (NSF) that will operate the instrument.

Associated Universities Incorporated (AUI), the contractor that operates the NRAO for the NSF, is locked in dispute with Comsat over who should pay the extra costs that arose during the project's ten-year construction (see Nature 384, 505; 1996).

Senator Byrd, who earmarked $\$ 75$ million of the NSF's budget to build the telescope in 1989, is thought to have favoured a dedication ceremony before November, when he stands for election to an eighth term in the US Senate.

The NRAO expects to take control of the telescope late next month. "I'm extremely hopeful that we'll have first light by the end of the year," says Phil Jewell, site director at Green Bank.

The GBT is of potential use to radioastronomers interested in everything from star formation to astrochemistry. But although pulsar astronomers are likely to start their observations next spring, the GBT

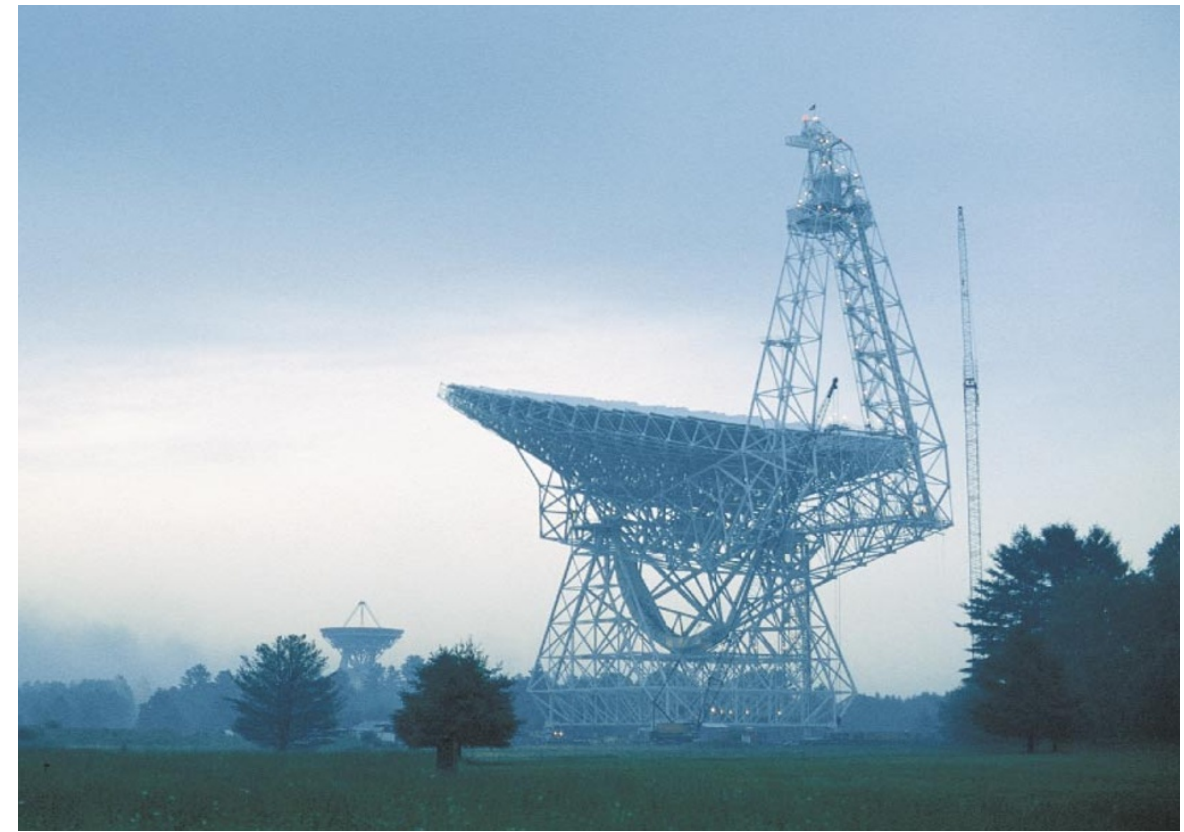

Big spender: the Green Bank Telescope was completed six years late and millions over budget.

will only come into its own for many other astronomers if it can perform at higher radio frequencies of around 100 gigahertz, corresponding to wavelengths of 3 millimetres. At these frequencies the telescope will, for example, be able to detect a number of molecular tracers and so provide new insight into the early behaviour of young stars.

But high-frequency work will require the successful commissioning of the instrument's laser-based metrology system. This is supposed to control the position of its panels to within 25 micrometres, using a feedback loop of 12 ground-based lasers and the 2,209 motorized actuators on which the panels are mounted.

"This has never been tried before," admits Jewell. "But all of the hardware has been built now, and the results of our ground-based tests are very encouraging. I am very confident it will work."

The commissioning process will be complicated by fiscal constraints at the NRAO, whose budget next year will be frozen at $\$ 32$ million - even assuming that the US Congress allows the NSF the budget it has requested for 2001 .

The 2002 House Appropriations Bill for the NSF contains language encouraging the agency to provide more funds for astronomy facilities, but does not actually provide any extra money. Robert Dickman, head of radioastronomy facilities at the NSF, says the agency's budget allows NRAO to compete for additional funds in areas such as information technology. "All the stakeholders are disappointed at the amount of time it has taken" to complete the telescope, he adds.

The budget crunch at the NRAO might get worse when the AUI/Comsat arbitration process is completed. The cost overruns that are currently under dispute amount to $\$ 28$ million, according to Paul Vanden Bout, director of the NRAO, although some sources put the number even higher. Arbitration will end in October and a ruling is expected some time in 2001 — possibly allocating some of the costs to the observatory.

Engineers at the Green Bank site are still wrestling with several challenges, such as devising and implementing a suitable maintenance programme for the telescope's surfaces, welds and actuators. Vanden Bout is optimistic that this can be done. "We know what we have to do," he says.

But some astronomers worry that the NRAO is more fully committed to other projects, such as the planned Atacama Large Millimeter Array (ALMA) in Chile and the expansion of the Very Large Array (VLA) in New Mexico.

Asked if the NRAO was committed to the GBT as central to its future, Riccardo Giacconi, president of Associated Universities Incorporated, said: "We've got three of four major facilities, including the VLA and ALMA. GBT is something that we are committed to turn from a technological success to a scientific success." He concedes, however, that this will be "a tough job".

It's also a job that may be the swansong for efforts to scale up the fully steerable, singledish radiotelescope, according to Taylor. "The trade-offs between a single large telescope and an array are such that it is not very likely that we'll want to build something that is even larger and fully movable," he says.

http://www.gb.nrao.edu/ 